\title{
Dyoram's Representation Using a Mosaic Image
}

\author{
${ }^{1}$ Juarez City University, Juarez City; Mexico \\ ${ }^{2}$ ISTC-CNR, Rome; Italy \\ ${ }^{3}$ Center of Research on Mathematics, Guanajuato; Mexico \\ ${ }^{4}$ CIATEC Center of Research CONACYT, León; Mexico \\ ${ }^{5}$ Veracruzana University, Xalapa; Mexico \\ ${ }^{6}$ Artificial Intelligence Laboratory, UAA, Aguascalientes; Mexico \\ ${ }^{7}$ Technological University of Zilina, Zilina; Slovak Republic
}

Alberto Ochoa ${ }^{1,2}$, Arturo Hernández ${ }^{3}$, S. Jöns ${ }^{4}$, Fernando Montes ${ }^{2,5}$, Julio Ponce ${ }^{6}$ and Lenka Janacek

\begin{abstract}
Evolving Optimization is a generic term used to make reference to the solution of planned and implemented computational problems with base in models of an evolutionary process. Most of the bioinspired algorithms, propose biological paradigms, and the concepts of natural selection, mutation, migration and reproduction or using other techniques are with a novel paradigm as Bacterial Foraging [8], which is based on only a small part in biology, and focus more on a branch of which this is biotechnology. Nevertheless, other paradigms exist that can be adopted in the creation of evolutionary algorithms. Many environment problems involve not structured knowledge, which can be considered from the perspective of cultural paradigms; the cultural paradigms offer an ample range of categorized models that do not know the possible solutions to the problem - a situation common in the real world.
\end{abstract}

The intention of the present research is to apply the computational properties of the cultural technology; in this case of corroborating of them by means of Social Data Mining to propose the solution to a specific problem, adapted from the Literature about Social Modelling. Combined with this, we analyzed the location of a society represented with its starship with respect to the social and cultural similarity of its neighbors, in a form of popular representation denominated Diorama. The set of study conformed by 95 societies (each society with a starship documented in [3]) allowed to analyze the social characteristics without affecting the extreme total of the sense of the Mosaic Image which represents the Diorama (what this it represents of all of them), and gave the opportunity us to emulate the distances that separate to each one of them and as these are grouped with respect to cluster that they belong. Demonstrating that ideological, social and cultural characteristic exist whose approximate them and as well they move away them. By means of this information it is possible to predict the best visualization of a Mosaic Image which represent a diorama, redistributing to the starships that conform it, this paper tries to explain this representation of social behavior.

Index Terms-Pattern Recognition, Image Mosaic and Social networks

\section{INTRODUCTION}

The Cultural Algorithms are an approach of Evolutionary

Manuscript received on 25 March 2008

E-Mail: megamax8@hotmail.com
Compute that uses the culture like a vehicle to store excellent and accessible information to all the members of the population during many generations. Like in a human society, the cultural changes act as advances the time, this one provides a line bases for the interpretation and documentation of individual behaviors within a society [1]. The Cultural Algorithms were developed to model the evolution of the cultural component on the time and to demonstrate how this one learns and acquires knowledge. In agreement with this conception, the cultural algorithms can be used to lead the process of the self-adaptation within evolutionary systems in a variety of diverse areas of application [4]. The cultural algorithm base can be described by means of the following pseudo code.

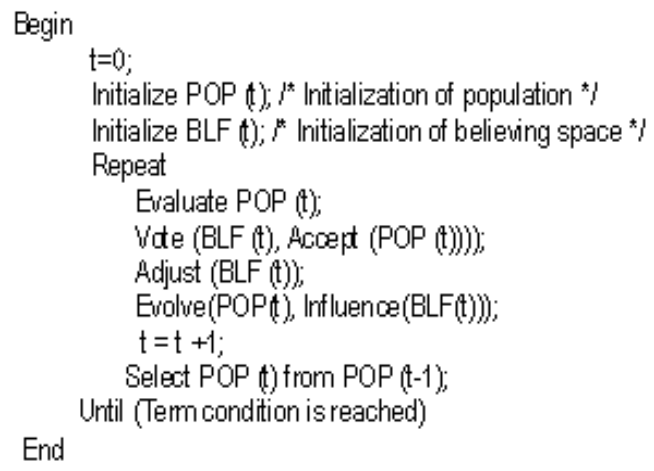

Fig. 1. Pseudo code base of Cultural Algorithms

Initially a population of individuals that represents the solution space, which is represented like a set of solutions within the space search, is generated randomly to create the first generation. In our example, the solution space contains a list of the attributes that can be used in the classification and location procedure. The space of beliefs is emptiness. For each generation, the Cultural Algorithm will be able to involve a population of individuals using "frame" Vote-Inherit-Promote (VIP). During the phase of Vote of this process, the members of the population are evaluated to identify their contribution to the space of beliefs being used the acceptance function. These beliefs allow contributing in most of the solution of the problem and are selected or put to voting to contribute to the present space of beliefs. The belief space is modified when the inherited 
beliefs are combined with the beliefs that have been added by the present generation, this is made using a reasoning process that allows updating the space of beliefs. Next, the space of beliefs updated is used to influence in the evolution of the population. The belief space is used to influence on the rest of the population and the acceptance of its beliefs is modified. During the last phase a new population is reproduced using a basic set of evolutionary operators. This new population could be evaluated and the cycle continues successively, until all the population has the same space of beliefs [5]. Cycle VIP finishes when a condition of completion is introduced. The condition of term usually is reached when only a small change or no one is detected in the population through several generations or when certain knowledge in the space has emerged from beliefs, as it is possible to be appreciated in Figure 2 is show a conceptual diagram which explain its components.

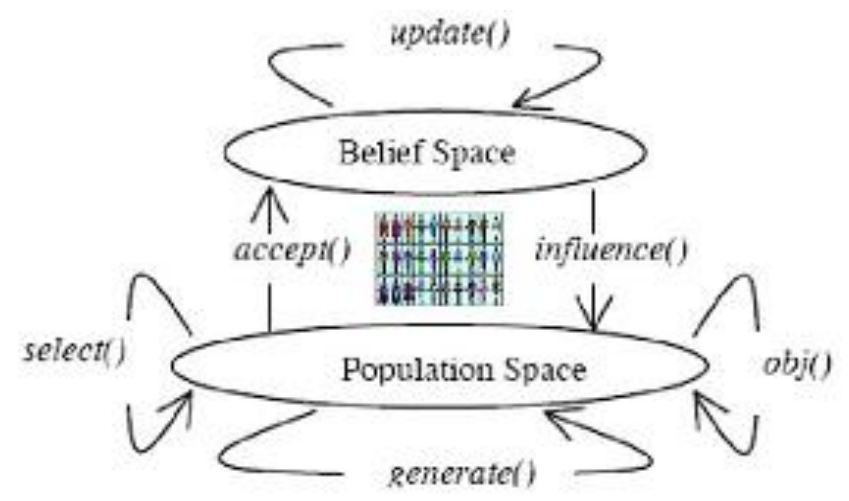

Fig. 2. Pseudo code base of Cultural Algorithms

\section{DYORAM: THE SOCIAL NETWORKING REPRESENTATION}

A social network is a social structure that can be represented making use of different types as diagrams. Both more common types are the graph and diorama. The graph is a collection of called objects vertices or nodes that are connected by called lines edges or arcs. The nodes represent individuals which sometimes are denominated actors and the edges represent the relations that exist between these actors. The relations can be of different type, like financial interchanges, sexual friendship, relations, or places of tourism. The social networks can be classified in: Dyadic: They only indicate absence or existence of the relation between two actors. Valued: In that the amount of the relation can be moderate in terms of order or weight like, for example, number of sexual encounter between two people. Transitive: The relation between an actor To and one B is always reciprocal. (Example: Both read the same Blog habitually.) Directed: In this case an actor To has relation with an actor B, but, that does not imply that B has the same relation with A. (Example: To lend money) the representation of a social network can consist of one or more graphs where these graphs conceptualizing the network, that is to say, the representation is made mainly on the basis of the relations that exist between the actors who conform the network. On the other hand dioramas are representations elaborated with materials or elements in three dimensions, which sometimes represent a scene of the real life. They are located in front of a curved bottom that can so be painted of way that it simulates real surroundings and can be completed with illumination effects. In this article, we focused our attention in a practical problem of the Literature related to Social Modelling, the accomplishment of a diorama, which allows to understand the position that keeps a society with respect to others, the capacity to establish the locations in the Diorama, allows to establish "the negotiation of the best location" for the given set of societies. The solution to this problem could be given by a sequence of generations of agents, denoted like "community". The agents can only reassigned a location with respect to the other societies, determined this according to previous behaviors, in each one of them [2]. In diorama can be represented: animals, plants, people, and battles among other things. In the case of the social networks, dioramas, unlike the graphs, characterizes the social network, that is to say, in diorama one of the actors who conform the network according to their roll and to the location that show at each one, in our case the starships represented the potential capability and cultural ranking. The development of the social networking requires on the one hand, of the conceptual development, and by another one, of the establishing of measures of mathematical discreet that allow ontologically exploring the human systems from a sustained concept and in the data. But it is necessary to prioritize the conceptual development and of the categories of the system of the social network, and parallel to think about the mathematical model, in our case using a Similarity Model which permits positions ubiquities of each society with respect at others. The profiles of each society are stored and with them we can compute the range between the rest of societies using theirs technological and cultural characteristics (to look for the similarities) and in this way of specifying the limits of clusters (Societies with similar preferences). We use the similarity function used in Social Data Mining to organize the societies in different clusters

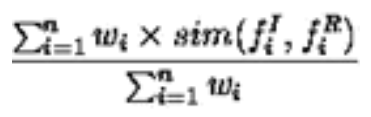

Where:

$\mathrm{W}_{\mathrm{i}}$ stands for weight of importance about an attribute. sim is the function of similarity.

$f_{i}^{I} y f_{i}^{R}$ are the values of attribute $i$ in the entrance cluster (I) and the recover cluster $(\mathrm{R})$.

\section{DISTRIBUTING ELEMENTS WITHIN A DYORAM}

From the point of view of the agents, this problem of optimization is very complex, on account that the best location of a representing starship of a society is not known, with respect to the other representatives. In the algorithm proposed for the cultural change, the societies in the space of beliefs (beliefscape) through their better paradigm (BestParadigm) are put to zero to represent the fact that the culture increases the amount of expectations associated with the location of a society with respect to the others, giving an incentive to the behavior associated with the best paradigm (BestParadigm). For it we 
selected 95 societies described in [3] and characterized their social behavior with base in seven social values: productivity, prosperity, creativity, spirituality, authority, knowledge, and support at other societies (solidarity), these attributes are used in the online game SIM Societies [10], these characteristics allow to describe so much to the society as to the individuals, for obtain better results, we programming a prototype in JBuilder which uses an image of a starship related with a society, the results is an Image Mosaic that contents all societies and its distribution in the Diorama (see figure 3). The development of the tool this based on our desire to share the intuitive understanding about the treatment of a new class of systems, societies able to have empathy, a reserved characteristic associated with people alive.

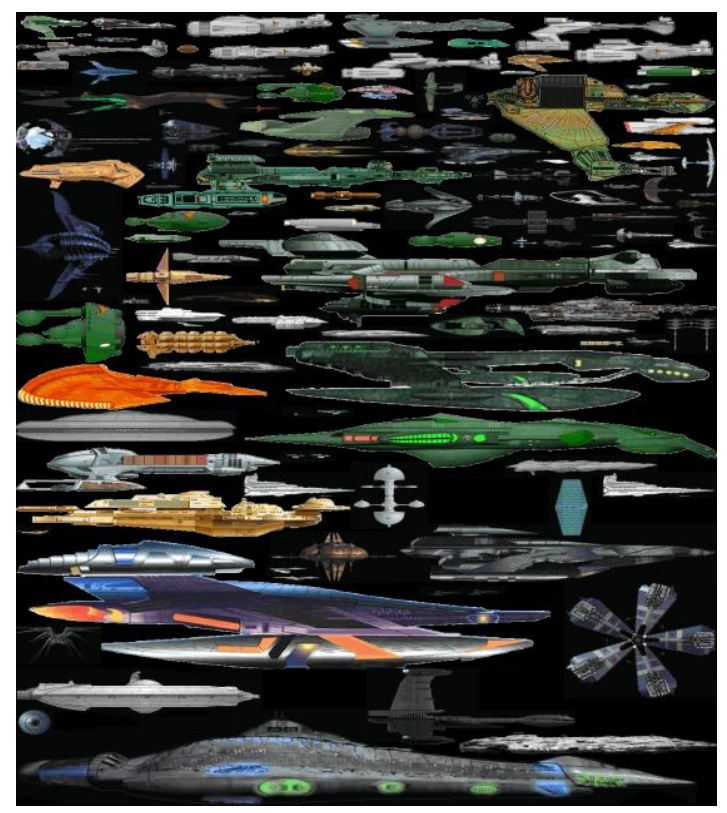

Fig. 3. Image Mosaic obtained means Cultural Algorithms represent 95 Societies [3] and its respective starships

\section{EXPERIMENTS}

In order to be able similar the most efficient arrangement of societies in a social network, we developed an environment able to store the data of each one of the representing starships of each society, this with the purpose of distributing of an optimal form to each one of the evaluated societies. One of the most interesting characteristics observed in this experiment was the diversity of the cultural patterns established by each community. The structured scenarios associated with the agents cannot be reproduced in general, since they only represent a little while dice in the space and time of the different societies. These represent a unique form and innovating of adaptive behavior which solves a computational problem that it does not try to clustering the societies only with a factor associated with his external appearance (features of each starship), trying to solve a computational problem that involves a complex change between the existing relations. The generated configurations can be metaphorically related to the knowledge of the behavior of the community with respect to an optimization problem (to conform to cluster culturally with other similar societies, without being of the same quadrant [3]). The main experiment consisted of detailing each one of the 95 communities, with 500 agents, and one condition of unemployment of 50 generations, this allowed us to generate different scenarios for to obtain a best possible diorama, which was obtained after comparing the different cultural and social similarities from each community, and to determine the existing relations between each one of them, the Mosaic Image resultant is the best distribution of all societies under these parameters. The developed tool classified each one of the societies pertaining to each quadrant, with two tonalities, the strong tone for societies that included technological identity and a smooth tone for societies only with cultural identity, this permit identify changes in the time respect at other societies (See Figure 4).

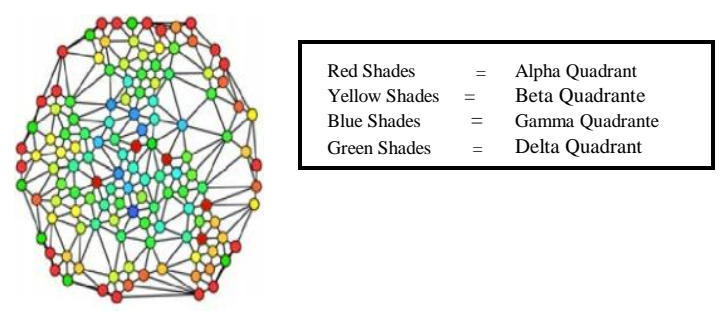

Fig. 4. Graph constructed by means of the use of Cultural Algorithms and Clusterization, support the Image Mosaic.

\section{CONCLUSIONS}

Using Cultural Algorithms we improved the understanding substantially to obtain the change of "best paradigm", because we appropriately classified the agent communities basing to us on an approach to the relation that keep their attributes, this allowed us to understand that the concept of "negotiation" exists with base in the determination of the function of acceptance, understanding with the knowledge from the rest of communities to the propose location for the rest of the similar societies. The Cultural Algorithms offer a powerful alternative for optimization problems and redistribution of clusterization. For that reason, this technique provides a quite comprehensible panorama with the cultural phenomenon represented [6]. This technique allowed us to understand the possibility of generating experimental knowledge created by the community of agents for a given dominion of application, such the location of many societies of four different Quadrants explained in [3]. The analysis of the level and degree of cognitive knowledge of each community is an aspect that is desired to evaluate for the future work. The answer can reside between the similarity that exists in the communication between two different cultures and as these are perceived for others. On the other hand to understand the true similarities that have different societies with base in the characteristics that make them contributor of cluster and it as well allows its to keep his own identity, demonstrates that the small variations go beyond technological characteristics, the starships represented different level of technology and are mainly associate to tastes and similar characteristics developed through the time [7] 


\section{ACKNOWLEDGMENT}

To Social Data Mining community which during a long time has researched on new ways to visualize social networking. The first author wishes thanks to Dayanara Zurutuza (Graduate Student) who implemented the prototype which generates the image mosaic to represent the 95 societies from Memory Alpha, as final project for a Bioinspired Algorithms course.

\section{REFERENCES}

[1] A. Desmond and J. Moore. "Darwin - la vida de un evolucionista atormentado". Generación Editorial, São Paulo, Brazil,1995.

[2] A. Maffucci. "Resolución de un Ridgelet Difuminado usando un algoritmo bioinspirado". CAEPIA'2005; Spain,2005.

[3] Memory Alpha. memory-alpha.org (Star Trek World),2007.

[4] Z. Hiramdóttir and A. Ochoa. "Optimization Problem Solving using Predator/Prey Game and Cultural Algorithms" NDAM'2003, Reykiavik; Iceland,2007.

[5] Tang Hué et al. "The Emergence of Social Network Hierarchy Using Cultural Algorithms", VLDB'06, Seoul,2006

[6] Zuckermann Dennis. "Culture and Organizations", London: McGrawHill, 1991.

[7] D. Đraguljović and A. Ochoa. "Similar cultural relationships in Montenegro" JASSS'2005, England.,2005

[8] Mezura Efrén and Hernández Betania. "Bacterial Foraging for Engineering Design Problems: Plemiminary Results", (COMCEV'2008,2008

[9] Caravelo, Francesco, "Ambient Temperature Modelling through Traditional and Soft Computing Methods", (HAIS'2008M. Young, The Technical Writer's Handbook. Mill Valley, CA: University Science, 1989,2008 .

[10] Ochoa, A. et al "Improve a Game Board based on Cultural Algorithms", The International Journal of Virtual Reality, 2008, 7/2):41-46,2008.

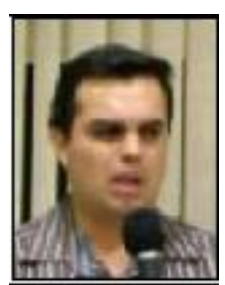

\section{Alberto \\ Ochoa \\ Ortiz-Zezzatti}

(Bs'94-Eng.Master'00;PhD'04-Postdoctoral Researcher'06 \& Industrial Postdoctoral Research'09). He joined the Juarez City University in 2008. He has 1 book, and 7 chapters in books related with AI. He has supervised $8 \mathrm{PhD}$ theses, $11 \mathrm{M} . \mathrm{Sc}$. theses and 27 undergraduate theses. He participated in the organization of COMCEV'07, COMCEV'08, HAIS'07, HAIS'08, HAIS'09, ENC'06, ENC'07, ENC'08 and MICAI'08. His research interests include evolutionary computation, natural processing language, anthropometrics characterization and Social Data Mining. In his second $\mathrm{PhD}$ participated in an internship in ISTC-CNR in Rome, Italy.

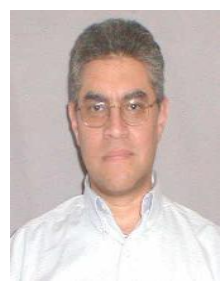

Arturo Hernández Aguirre is a researcher and professor in the Computer Science Department of the Center for Research in Mathematics (CIMAT). His research interests are Multiobjective Optimization, Evolvable hardware, Evolutionary computation, Evolutionary design of neural network architectures and Computational Learning Theory using neural networks.

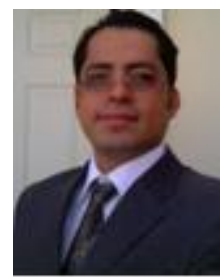

S. Jöns (Bs'00-Eng.Master'02 both from Instituto Tecnológico of Querétaro and PhD'07 from CIATEC-Conacyt Research Center). His research field is the mathematical modelling and optimization of process and products using response surface methodology and genetic algorithms. Furthermore, he is a professor of the postgraduate program of CIATEC.

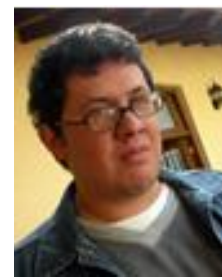

Fernando Montes $\mathrm{He}$ obtained his degree in Information Technology from the University of Veracruz in his hometown, Xalapa, which is in Veracruz, Mexico. His degree focused on the study of Information Systems related to the business field. He has some experience in working as IT technician, as $\mathrm{He}$ has done some work of this kind. After having applied to a scholarship from the Mexican government (CONACyT), He did a Master's Degree on Artificial Intelligence at the University of Veracruz. After starting his Master's dissertation his research interest started to develop, and He participated in a project called Monots (Monkey-Robots). The aim of this project was the simulation of foraging behavior in the Howler Monkey (Alouatta Palliata). And as part of the same project He worked with the Maes' model of action selection (Behavioral Networks) for carrying out the mentioned simulation. Later, thanks to a scholarship granted by CONACyT, He finished his Ph.D. at the University of Sheffield in the area of Artificial Intelligence. The name of his dissertation was: "A Robot Model of Action Selection in the Vertebrate Brain" and is related to animal behavior, reactive robotics and neuroscience. Among his research interests is to keep working in the area of AI and neurosciences. His research interests mainly focus on Artificial Intelligence, Behavior Based Robotics, Action Selection Mechanisms, Genetic Algorithms, and recently Neuroscience, specifically the role of basal ganglia in Evolutionary Action Selection.

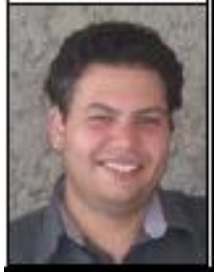

Julio Ponce (Bs'03- Master '07). He is currently working towards the $\mathrm{PhD}$ degree in eveolutionary computation from the UAA. He is currently Professor in the Universidad Autónoma de Aguascalientes. His research interests include Evolutionary Computation and Data Mining.

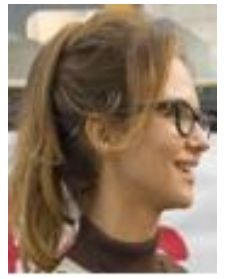

Lenka Janacek (BS '95-Master'99-PhD'04). She obtained a PhD from Universitá La Sapienza in Roma, Italy with a scholarship from RAI Internazionale. She realized in 2006 an Intership in Aächen University in Germany; actually she has many papers related with Artificial Intelligence in the developing of Artificial Societies. She participated in the Technical Committee of HAIS'09. Her research interests include Hybrid Artificial intelligent Systems. 